Gestión de la producción

Production Management 



\title{
Gestión de mantenimiento preventivo y su relación con la disponibilidad de la flota de camiones 730e Komatsu-2013
}

\author{
Carol Alavedra Flores*, Yumira Gastelu Pinedo*, Griseyda Méndez Orellana*, Christian \\ Minaya Luna*, Brandon Pineda Ocas*, Krisley Prieto Gilio*, Kenny Ríos Mejía* \\ Universidad César Vallejo. Chimbote, Perú \\ César Moreno Rojo* \\ Universidad Nacional del Santa. Chimbote, Perú
}

Recibido: 30 de mayo del 2016 / Aprobado: 10 de julio del 2016

\begin{abstract}
Resumen: Todo sistema es productivo, siempre y cuando opere bajo un mínimo de fallas. Basada en este principio, la investigación en la empresa Komatsu Maquinarias Perú S. A. consistió en el análisis de la situación actual de los equipos y determinó cuál es la relación entre la gestión de mantenimiento preventivo a través de sus indicadores y la disponibilidad. Realizado el análisis, el coeficiente de correlación es 79,1\%, lo que nos indica que existe un regular grado de relación entre las variables de disponibilidad MTBF y MTTR.
\end{abstract}

Palabras clave: gestión / mantenimiento / disponibilidad / camiones / Komatsu maquinarias Perú S. A

\section{Preventive maintenance management and its relationship to the availability of the fleet truck 730e Komatsu-2013}

ABSTRACT: All production system is provided to operate under minimal of faults. Considering this, the research in the company Komatsu Machinery Peru S.A., it was an analysis of the current status of the equipment and determine the relationship between preventive maintenance management through its indicators and availability. Performed the analysis, the correlation coefficient is $79,1 \%$. This indicates that there is a regular degree of relationship between the variables availability MTBF and MTTR.

Keywords: management / maintenance / availability / trucks / Komatsu machinery Perú S. A

* Correos electrónicos: carol_ale21@hotmail.com, yumiragastelu@hotmail.com, virgo.angelix_15@hotmail.com, minaya_12_96@hotmail.com, brax_tkm@hotmail. com, krisleypg@gmail.com, kenny_2_02@hotmail.com, cemoro67@yahoo.es 


\section{CONCEPTOS GENERALES DEL MANTENIMIENTO PREVENTIVO Y LA DISPONIBILIDAD}

Al mantenimiento preventivo se le puede definir como la conservación planeada. Tiene como función permitir el conocimiento sistemático del estado de las máquinas y equipos para programar la tarea que debe realizarse, en los momentos más oportunos y de menor impacto.

El mantenimiento preventivo se refiere a que no se debe esperar a que las máquinas fallen para hacerles una reparación, sino que se programen los recambios con el tiempo necesario antes de que se presente la falla; esto se puede lograr conociendo las especificaciones técnicas de los equipos a través de los manuales ${ }^{1}$.

Si consideramos que la empresa Komatsu realiza trabajos en el sector minero y de la construcción, para lo que se requiere de maquinaria pesada y especializada para el desarrollo de los procesos de carguío, el desgaste que sufre este tipo de equipos y herramientas es un aspecto relevante que la empresa tiene en cuenta durante todo el proceso. Para prevenir paralizaciones de las máquinas en pleno funcionamiento, la empresa realiza un análisis previo o mantenimiento para que se detecten fallas y sean reparadas en el tiempo necesario.

La finalidad del mantenimiento preventivo es encontrar y corregir los problemas menores antes de que provoquen fallas. El mantenimiento preventivo también puede ser definido como una lista completa de actividades, todas realizadas por usuarios, operadores y encargados de mantenimiento, para asegurar el correcto funcionamiento de la planta, edificios, máquinas, equipos, vehículos, etc. ${ }^{2}$ De esta manera, se tendrá la confiabilidad de que estos equipos operen en adecuadas condiciones de seguridad, ya que se conoce su estado y niveles de funcionamiento, consiguiendo una disminución del tiempo muerto, menos existencias en almacenes y, por ende, la reducción de los costos.

1 Vargas, P. y Welder, U. (2004). Programa de mantenimiento para la maquinaria pesada de la zona vial 11, de caminos, en el Departamento de Izabal (Tesis de Ingeniería Mecánica). Universidad de San Carlos de Guatemala, p. 50.

2 Báez, A. y Caraballo, R. (2004). Desarrollo de un plan de mantenimiento para una industria textil basado en mantenimiento productivo total utilizando un sistema computarizado (Tesis de Ingeniería Mecánica) Universidad Central de Venezuela, Caracas, p. 50. 
No podemos dejar de relacionar el mantenimiento preventivo con la disponibilidad, pues son dos variables que van de la mano. Por lo tanto, debemos resaltar que la disponibilidad es el trabajo completo que se le da a un equipo y la confiabilidad que este tiene en la operación. La disponibilidad de un equipo representa el tiempo disponible de un sistema al servicio de la unidad de producción, la cual se calcula en porcentaje en un tiempo determinado. Según Knezevic, la disponibilidad es una característica que resume cuantitativamente el perfil de funcionabilidad de un elemento ${ }^{3}$.

La mayoría de los usuarios afirman que se necesita la disponibilidad del equipo tanto como la seguridad, porque no se puede tolerar tener un equipo fuera de servicio. Hay varios medios para lograrlo. Uno es construir los equipos extremadamente fiables y, consecuentemente, más costosos. El segundo es suministrar un sistema que, aunque falle, sea fácil de recuperar. De esta forma, si todo está construido muy fiablemente y es fácil de reparar, el fabricante obtiene un sistema muy eficaz, pero que aumenta considerablemente su valor.

El factor de disponibilidad de un equipo o sistema es una medida que nos indica cuánto tiempo está funcionando ese equipo o sistema operativo respecto de la duración total durante el periodo en el que se desea que funcione. Típicamente se expresa en porcentaje, no debe ser confundido con la rapidez de respuesta.

La disponibilidad de un equipo solo puede aumentarse disminuyendo el tiempo fuera de servicio, lo cual es posible con la mejora de los sistemas administrativos, los procedimientos, la selección, el entretenamiento, la motivación del personal, la calidad y dotación de herramientas, el equipo de diagnóstico, los sistemas de información de equipos y la optimización de los sistemas de abastecimiento. Por lo tanto, se requiere de herramientas que permitan tomar decisiones rápidas y acertadas sobre sus principales recursos, que para las operaciones de movimiento de tierras involucre directamente a los equipos.

Asimismo, una empresa se crea con la finalidad de obtener utilidades, lo que está directamente ligado a los plazos en el tiempo de cada una de sus actividades. Por ello, es necesario tener una herramienta de control que nos brinde una fotografía del estado del negocio en un momento determinado (venta vs. costo), y que facilite tomar decisiones

3 Knezevic, J. (1996). Mantenimiento, (1. ${ }^{a}$ ed.). España: Isdefe, p. 23. 
a tiempo para cumplir las metas trazadas. Por consiguiente, es indispensable contar con una planificación técnica y formal, que disminuya las probabilidades de falla o presentación de imprevistos que conlleven a no cumplir las metas. En vista de esta necesidad, el presente artículo busca demostrar una metodología práctica y económica, para planificar y controlar los requerimientos del equipo pesado que se maneja dentro de la empresa, dándole a esta la disponibilidad mecánica que requiere para la optimización de sus operaciones.

El estudio a desarrollar fue del tipo descriptivo correlacional, pues plantea respuestas a la relación entre la gestión de mantenimiento preventivo y la disponibilidad. Se tuvo como propósito medir la relación entre ambas variables en un contexto particular, para analizar posteriormente su causalidad y efecto.

Con esta finalidad se realizaron visitas al taller, entrevistas no estructuradas, reuniones con los mecánicos líderes del taller y con los inspectores de los equipos mecánicos en estudio.

\section{ANÁLISIS DE LA GESTIÓN DE MANTENIMIENTO PREVENTIVO}

Para el desarrollo del análisis de la gestión de mantenimiento preventivo se tomó en cuenta la información obtenida de datos históricos de la flota de camiones 730e Komatsu, mediante los indicadores MTBF y MTTR. A partir de ello, se observó el comportamiento de la disponibilidad a través del tiempo y cómo la gestión del mantenimiento ha influido en el buen desarrollo del estado de los equipos y su disponibilidad.

Tras realizar el análisis de las figuras 1 y 2 , se observó que el indicador del MTBF (tiempo medio entre fallas) mostró un descenso progresivo y sostenido durante el tiempo. Por ende, mientras aumenta el tiempo, también se incrementan las paradas inopinadas por mantenimiento correctivo.

El MTBF del año 2011 tuvo una caída con respecto al año anterior, aproximadamente de 9,68 \%, con una varianza de 11,59 horas. Se aprecia que el MTBF del año 2012, en comparación al del año 2011, toleró una caída de 20,20 \%, con una varianza de 21,83 horas. Asimismo, el MTBF del año 2013, con respecto al año 2012, sufrió una caída del $39,51 \%$, con una varianza de 34,07 horas. 
Tabla 1

Histórico de datos del MTBF (2010-2013)

Tiempo medio entre fallas (MTBF)

Flota Komatsu 730e

\begin{tabular}{cr|cr|cr}
\hline Mes & MTBF & Mes & MTBF & Mes & MTBF \\
\hline Abr-10 & 142,65 & May-11 & 138,87 & Jun-12 & 66,51 \\
May-10 & 167,4 & Jun-11 & 132,84 & Jul-12 & 56,88 \\
Jun-10 & 92,7 & Jul-11 & 78,3 & Ago-12 & 101,7 \\
Jul-10 & 65,52 & Ago-11 & 109,8 & Set-12 & 146,43 \\
Ago-10 & 102,42 & Set-11 & 103,23 & Oct-12 & 70,65 \\
Set-10 & 124,47 & Oct-11 & 67,41 & Nov-12 & 45,54 \\
Oct-10 & 97,38 & Nov-11 & 65,43 & Dic-12 & 91,98 \\
Nov-10 & 193,05 & Dic-11 & 77,85 & Ene-13 & 36,54 \\
Dic-10 & 91,35 & Ene-12 & 61,29 & Feb-13 & 40,23 \\
Ene-11 & 147,69 & Feb-12 & 58,86 & Mar-13 & 51,94 \\
Feb-11 & 84,42 & Mar-12 & 103,32 & Abr-13 & 55,98 \\
Mar-11 & 109,98 & Abr-12 & 63,63 & May-13 & 42,84 \\
Abr-11 & 92,61 & May-12 & 162,09 & Jun-13 & 65,25 \\
\hline
\end{tabular}

Fuente: Área de mantenimiento Komatsu Mitsui Maquinarias Perú S. A. (2010-2013)

MTBF de la flota de camiones $730 \mathrm{e}$

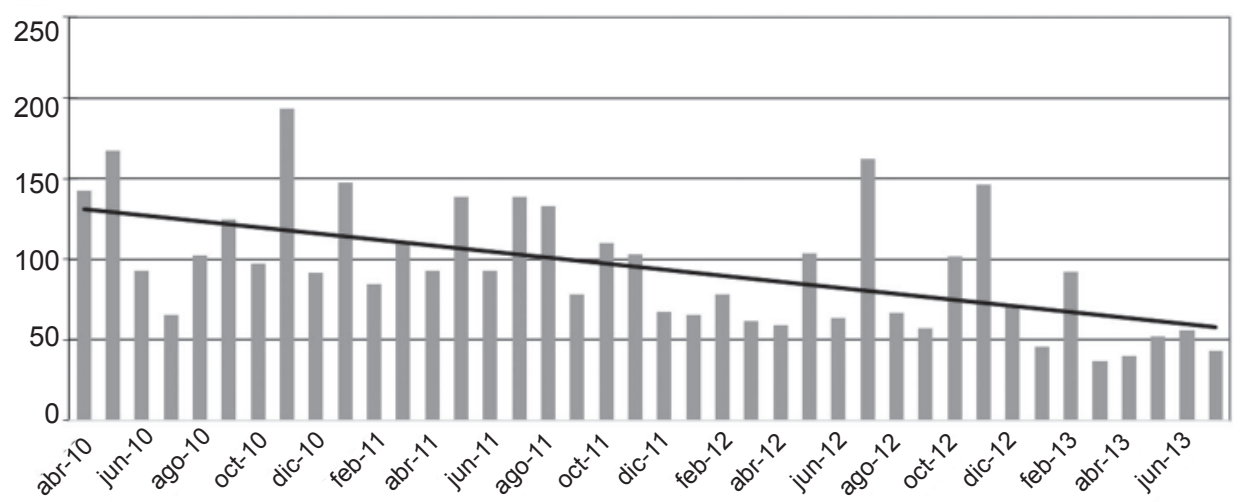

Figura 1. Tiempo medio entre fallas

Fuente: Área de mantenimiento Komatsu Mitsui Maquinarias Perú S. A. (2010-2013)

Elaboración propia 
MTBF de la flota de camiones 730 e

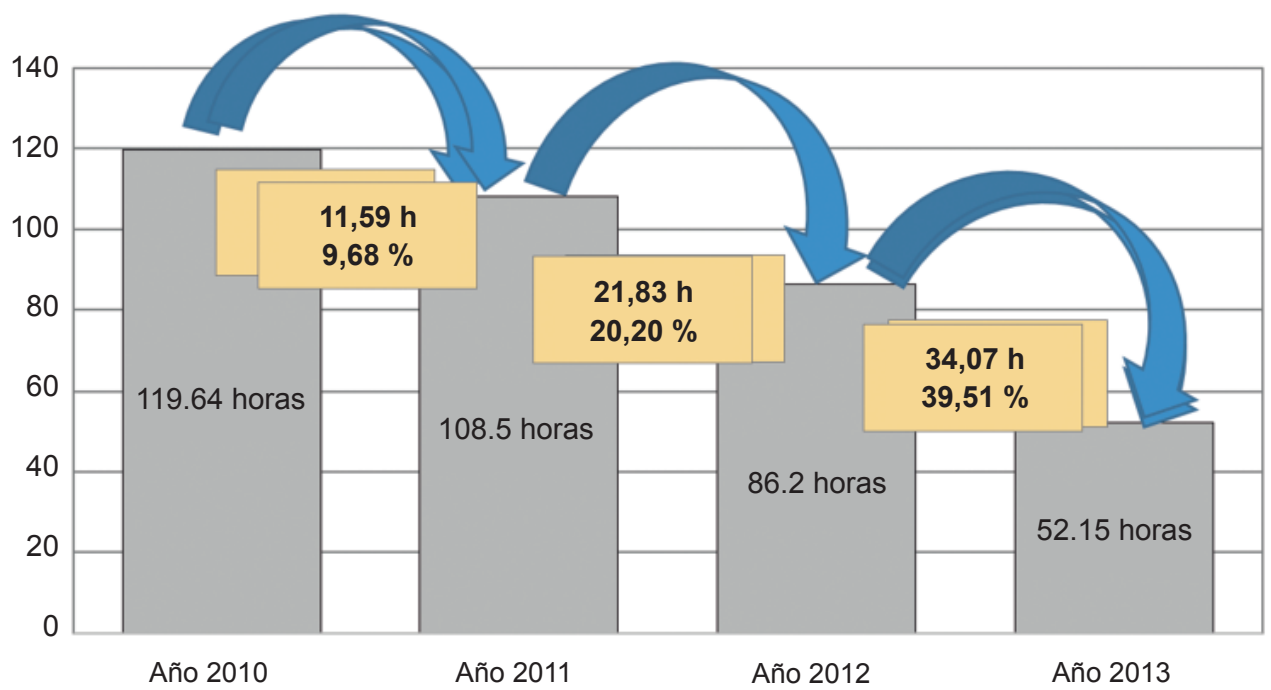

Figura 2. Tiempo medio entre fallas (2010-2013)

Fuente: Área de mantenimiento Komatsu Mitsui Maquinarias Perú S. A. (2010-2013)

Elaboración propia

Según el análisis del MTBF, la línea de tendencia expresa una caída en el tiempo si se continúa con el proceso.

Las paradas inesperadas dadas por el MTBF en la flota de camiones de Komatsu Mitsui indican que se tendrá que parar la producción para poder hacer el mantenimiento correctivo. Esas paradas generan un costo para la empresa, por lo cual esos errores tendrán que subsanarse haciendo un mantenimiento preventivo.

A continuación, se detallan los datos históricos del tiempo medio para reparar (tabla 2).

Con los datos de la tabla 2 se procedió a realizar la gráfica de tendencia para observar el comportamiento del MTTR y realizar el análisis, como la mostramos en la figura 3.

Analizando la figura 3 , se observa que existe una tendencia progresiva del tiempo medio para reparaciones, es decir, que los tiempos de las reparaciones son progresivamente crecientes y que los problemas se agravan cada día más. 
Tabla 2

Histórico de datos del MTTR del 2012-2013

\begin{tabular}{cc}
\hline \multicolumn{2}{c}{ Tiempo medio para reparar (horas) } \\
\hline Periodo & MTTR \\
\hline Ago-12 & 3,10 \\
Set-12 & 1,58 \\
Oct-12 & 2,10 \\
Nov-12 & 1,90 \\
Dic-12 & 2,05 \\
Ene-13 & 3,20 \\
Feb-13 & 2,45 \\
Mar-13 & 1,85 \\
Abr-13 & 1,90 \\
May-13 & 1,75 \\
Jun-13 & 2,87 \\
Jul-13 & 3,20 \\
\hline
\end{tabular}

Fuente: Área de mantenimiento Komatsu Mitsui Maquinarias Perú S. A. (2012-2013)

MTTR de la flota de camiones $730 \mathrm{e}$

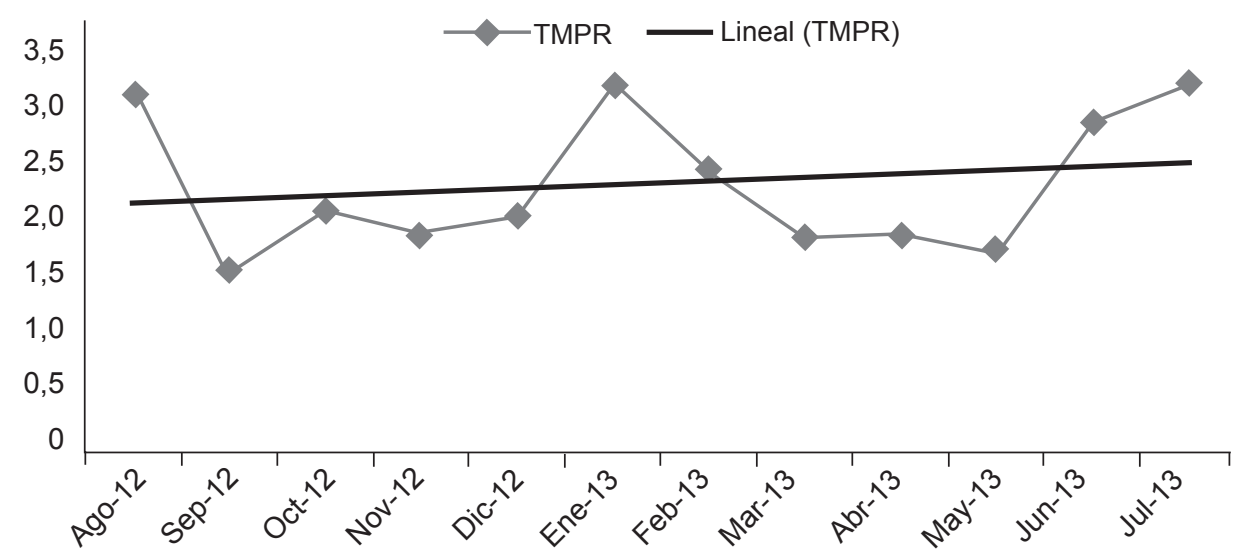

Figura 3. Tiempo medio para reparar Fuente: Área de mantenimiento Komatsu Mitsui Maquinarias Perú S. A. (2012-2013) Elaboración propia 


\section{ANÁLISIS DE LA DISPONIBILIDAD}

Los resultados obtenidos de la gestión de mantenimiento mensual de la disponibilidad física, tiempos medios entre fallas (MTBF) y tiempos medios para reparar (MTTR) se muestran a continuación. Fueron utilizados para determinar el modelo matemático y, de esa manera, relacionar la disponibilidad con la gestión de mantenimiento. Para ello, tomamos los datos de los años 2012 y 2013, que para nuestro estudio está dado por el último año.

Tabla 3

Histórico de datos de la disponibilidad física (2012-2013)

\begin{tabular}{cc}
\hline Periodos & Disponibilidad física \\
\hline Ago-12 & 84,6 \\
Set-12 & 88,2 \\
Oct-12 & 89,8 \\
Nov-12 & 90,7 \\
Dic-12 & 91,1 \\
Ene-13 & 90,3 \\
Feb-13 & 84,6 \\
Mar-13 & 89,5 \\
Abr-13 & 88,2 \\
May-13 & 89,1 \\
Jun-13 & 88,2 \\
Jul-13 & 86,0 \\
\hline
\end{tabular}

Fuente: Área de mantenimiento Komatsu Mitsui Maquinarias Perú S. A. (2012-2013)

Elaboración propia

$\mathrm{Al}$ analizar la figura 4, se observa que la disponibilidad tiene un descenso durante el tiempo, lo que nos indica que los mantenimientos preventivos no se están realizando de forma correcta y adecuada. Esto afecta la producción de extracción del material estéril (sin valor económico) que permite extraer, a su vez, el mineral útil que necesita el cliente. Por lo tanto, aumentan los costos. 


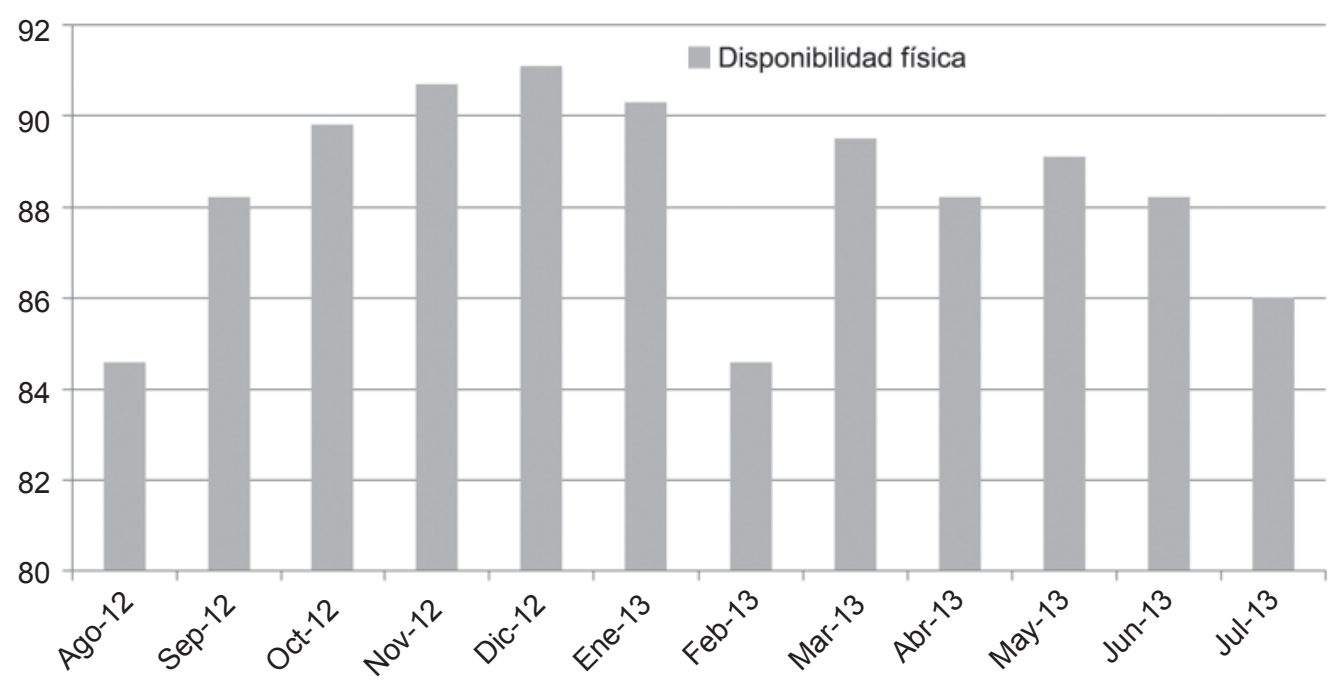

Figura 4. Disponibilidad física (2012-2013)

Fuente: Área de mantenimiento Komatsu Mitsui Maquinarias Perú S. A. (2012-2013)

Elaboración propia

\section{RESULTADO DEL ANÁLISIS DE LA GESTIÓN DE MANTENIMIENTO PREVENTIVO Y LA DISPONIBILIDAD}

\subsection{Análisis de los resultados de la regresión múltiple}

Luego de realizar el ingreso de los datos (tablas 1, 2 y 3) en el software de análisis de regresión múltiple (SPSS v20), se obtuvieron los siguientes resultados.

Tabla 4

Valores tomados para la formulación del modelo de regresión múltiple

\begin{tabular}{|c|c|c|c|c|c|}
\hline \multicolumn{6}{|c|}{ Coeficientes $^{a}$} \\
\hline \multirow[t]{2}{*}{ Modelo } & \multicolumn{2}{|c|}{$\begin{array}{l}\text { Coeficientes no } \\
\text { estandarizados }\end{array}$} & \multirow{2}{*}{$\begin{array}{c}\text { Coeficientes } \\
\text { tipificados } \\
\text { Beta }\end{array}$} & \multirow[t]{2}{*}{$\mathrm{t}$} & \multirow[t]{2}{*}{ Sig. } \\
\hline & $\mathrm{B}$ & Error típ. & & & \\
\hline 1. (constante) & 92,566 & 1,428 & & 64,824 & ,000 \\
\hline Tiempo medio entre fallas & 011 & 014 & ,160 & ,754 & ,470 \\
\hline $\begin{array}{l}\text { Tiempo medio entre } \\
\text { reparación }\end{array}$ & $-2,676$ & ,693 &,- 820 & $, 3,864$ & ,004 \\
\hline
\end{tabular}

Nota: a. Variable dependiente: Disponibilidad

Fuente: Área de mantenimiento Komatsu Mitsui Maquinarias Perú S. A. (2010-2013) 
Como se puede visualizar, la disponibilidad es la variable dependiente del MTTR y MTBF, dado que si se le aplica un mantenimiento preventivo a las máquinas, la disponibilidad que tendremos será favorable. De esa manera, los tiempos medios para reparar disminuirán y se obtendrá una mayor productividad y rendimiento.

El modelo de regresión múltiple ajustada del análisis para nuestro estudio está dado por:

$$
Y=\beta 0+\beta 1, X 1-\beta 2, X 2
$$

Ecu. 1

Luego, reemplazando los valores de la tabla 1 en $\beta 0 ; \beta 1 ; \beta 2$; tenemos que:

$$
Y=92,566+0,110 X 1-2,676 \times 2
$$

Ecu. 2

Por lo tanto, la función sería:

$$
Y=92,566+0,110(M T B F)-2,676(M T T R) \quad \text { Ecu. } 3
$$

Donde:

Y: disponibilidad

MTBF: tiempo medio entre fallas

MTTR: tiempo medio para reparar

\subsection{Análisis y resultado de la tabla de análisis de varianza (Anova)}

De acuerdo al análisis de la tabla de análisis de varianza (tabla 5), realizado en el programa de regresión múltiple (software SPSS v20), se obtuvo:

Tabla 5

Análisis de varianza

\begin{tabular}{lccccc}
\hline \multicolumn{5}{c}{ Anova $^{\mathrm{a}}$} \\
\hline Modelo & $\begin{array}{c}\text { Suma de } \\
\text { cuadrados }\end{array}$ & GI & $\begin{array}{c}\text { Media } \\
\text { cuadrática }\end{array}$ & F & Sig. \\
\hline 1 Regresión & 34,147 & 2 & 17,073 & 7,517 &, $012^{\mathrm{b}}$ \\
$\begin{array}{l}\text { Residual } \\
\text { Total }\end{array}$ & 20,443 & 9 & 2,271 & & \\
\hline
\end{tabular}

Nota: a. Variables dependientes: disponibilidad

b. Variables predictoras: (constante), tiempo medio entre reparación, tiempo medio entre fallas

Elaboración propia 
Donde:

- El grado de libertad (gl) para los puntos críticos es de $(2,9)$.

- El punto crítico de acuerdo a la tabla de Fisher para F(2,9) está dado por: $\mathrm{F}_{\text {crítico }}=4,257$ (valor dado por la tabla de valores críticos de la distribución F de Snedecor para un $\alpha=0,05)^{4}$.

- El valor de criticidad está dado por $\mathrm{F}=7,517$.

- El valor de significancia para el cálculo es: 0,012.

\subsection{Prueba de hipótesis en la regresión múltiple}

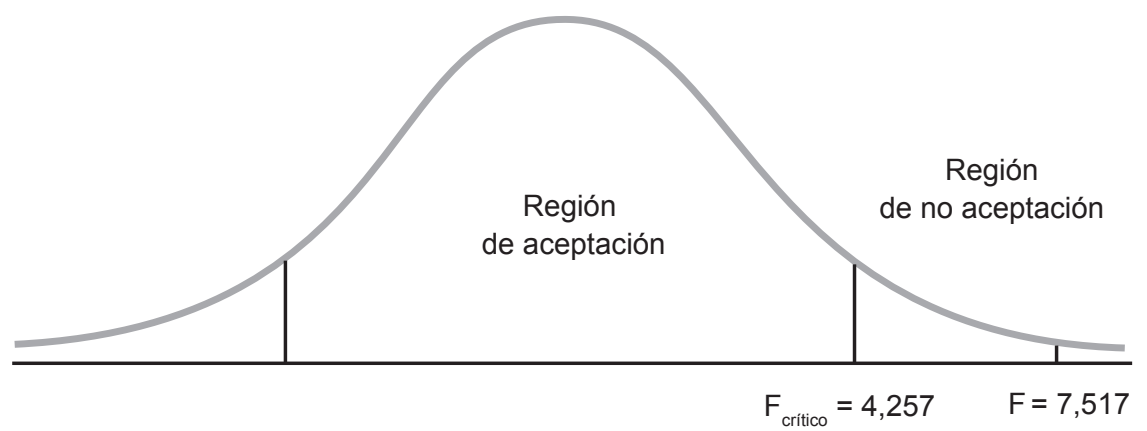

Figura 5. Campana de Gauss

Nota: Distribución F de Snedecor con grados de libertad 2 y 9

Elaboración propia

Donde:

R. A. = Región de aceptación y R. N. A. = Región de no aceptación

De acuerdo al análisis de la figura 5, tenemos que la estadística de prueba $\mathrm{F}$ (Fisher) está dada por $\mathrm{F}=7,517$, que es mayor que el punto crítico.

$\mathrm{F}_{\text {crítico }}=4,257$ (valor dado por la tabla de valores críticos de la distribución $\mathrm{F}$ de Snedecor para un $\alpha=0,05)$ para los grados de libertad de $(2,9)$ y cualquier nivel común de importancia. Tiene una significancia menor a 0,05 (Sign. < 0,05), por lo que la hipótesis nula es rechazada, y

4 Walpole R., Myers R. y Myers S. (1999). Probabilidad y estadistica para ingenieros (6. ${ }^{\mathrm{a}}$ ed.). México: Pearson. 
concluimos que la variable dependiente está relacionada con una o más de las variables independientes.

$$
\mathrm{H}_{0}=\beta_{1}=\beta_{2}=\beta_{3}=\ldots=\beta_{\mathrm{n}}
$$

Rechazar

Por lo tanto, se acepta la hipótesis alternativa.

$$
\mathrm{H}_{1}=\beta_{1}=\beta_{2}=\beta_{3}=\ldots=\beta_{\mathrm{n}} \neq 0
$$

Aceptar

Entonces, aceptamos la ecuación 3, dada por:

$$
Y=92,566+0,110(M T B F)-2,676(M T T R)
$$

Por lo tanto, con este modelo de regresión múltiple ajustado se pudo determinar valores adecuados para mantener un mejor margen de mantenimientos correctos, de tal manera que se pueda corregir todas las fallas en función de los indicadores dados.

4.4 Resultado del análisis del coeficiente de correlación múltiple (r) y del coeficiente de determinación múltiple $\left(r^{2}\right)$

Luego de aceptada la hipótesis de afirmación y de tener la ecuación lineal múltiple, se aplicó la fórmula para hallar los coeficientes, dados por:

Fórmula para hallar el coeficiente de correlación

Fórmula para hallar el coeficiente de determinación

$$
r=\frac{\sum_{i=1}^{n}\left(X_{i}-\bar{X}\right)\left(Y_{i}-\bar{Y}\right)}{\sqrt{\sum_{i=1}^{n}\left(X_{i}-\bar{X}\right)^{2} \sum_{i=1}^{n}\left(Y_{i}-\bar{Y}\right)^{2}}}
$$

Verificándose que $-1 \leq \mathrm{R} \leq 1$

$$
1-\mathrm{R}^{2}=\frac{S S E}{\sum_{i=1}^{n}\left(Y_{i}-\bar{Y}\right)^{2}}
$$

Para ello utilizamos el software SPSS v20, de acuerdo con los valores históricos presentados en el modelo. Se obtuvo lo siguiente: 
Tabla 6

Cuadro resumen del modelo

\begin{tabular}{ccccc}
\hline \multicolumn{4}{c}{ Resumen del modelo $^{\mathbf{b}}$} \\
\hline Modelo & $\mathrm{R}$ & R cuadrado & $\begin{array}{c}\text { R cuadrado } \\
\text { corregida }\end{array}$ & $\begin{array}{c}\text { Error típ. de la } \\
\text { estimación }\end{array}$ \\
\hline 1 &, $791^{\mathrm{a}}$ &, 626 &, 542 & 1,50712 \\
\hline
\end{tabular}

Nota: a. Variables predictoras: (constante), tiempo medio para reparar, tiempo medio entre fallas

b. Variable dependiente: disponibilidad

Elaboración propia

Del análisis de la tabla 6 , se obtiene que el coeficiente de correlación $r=0,791(79,1 \%)$. Esto nos indica que existe un considerable grado de relación positiva (ver tabla 1: Nivel de medición de variables) entre la variable dependiente (disponibilidad) y las independientes: MTBF y MTTR.

Luego, el coeficiente de determinación múltiple $\mathrm{R}^{2}=0,626(62,6 \%)$ indica que el $62,6 \%$ de la variabilidad en la disponibilidad se explica cuando se usan las dos variables de regresión MTBF y MTTR.

Por un lado, en la empresa minera Coimolache S. A. se realizó un estudio sobre el mantenimiento preventivo basado en la lubricación y la confiabilidad de las maquinarias, donde se hizo un diagnóstico de la gestión actual del sistema de lubricación. Encontramos los siguientes problemas: temperaturas de funcionamiento elevadas, fugas de lubricante, lubricante inadecuado y tiempo de demora para realizar las tareas de lubricación. Dentro de las principales causas de los problemas del sistema de lubricación actual, se hallaron: gestión de la lubricación, infraestructura, método y entrenamiento.

En la investigación hecha en la empresa Komatsu S. A. se realizó un estudio de la disponibilidad de los camiones Komatsu 730e, para plantear un modelo matemático a través de la regresión lineal múltiple. Determinamos el grado de relación entre la gestión del mantenimiento preventivo y la disponibilidad (62,6\%), y cómo este influirá en el buen desarrollo del mantenimiento para aumentar el nivel de disponibilidad en los equipos, que son los camiones 730e Komatsu. Para ello se han planteado las siguientes recomendaciones: determinar las causas 
del problema en el proceso; realizar un estudio de análisis de fallas y determinar las más críticas; implementar un plan de mantenimiento preventivo, con la finalidad de aumentar la disponibilidad y la confiabilidad de la flota de camiones 730e y disminuir sistemáticamente las paradas imprevistas de los equipos; disminuir los costos de inventarios y los costos de mantenimiento innecesarios.

Por lo tanto, se tiene que las variables de gestión de mantenimiento y disponibilidad tienen una relación de $62,6 \%$, lo que significa que el $37,4 \%$ de diferencia está dado por otros factores que no están relacionados con las variables. El coeficiente de determinación múltiple ajustado es de $\mathrm{R}_{\text {ajus }}^{2}=56,40 \%$.

Luego de realizar el análisis con los datos históricos del MTBF, MTTR y la disponibilidad de los camiones Komatsu 730e, se observó que hay una línea de tendencia decreciente, debido a los factores relevantes que afectan directamente al mantenimiento preventivo.

El MTBF del año 2011 tuvo una caída con respecto al año anterior, aproximadamente de $9,68 \%$, con una varianza de 11,59 horas. Se aprecia que el MTBF del 2012, en comparación con el del año 2011, toleró una caída de 20,20 \%, con una varianza de 21,83 horas. Asimismo, el MTBF del 2013 con respecto al del año 2012 sufrió una caída del $39,51 \%$, con una varianza de 34,07 horas. Por lo tanto, la línea de tendencia expresa una caída en el tiempo si se continúa con el proceso.

Se pudo observar que en el MTTR también existe una tendencia en ascenso, es decir, que los tiempos de las reparaciones se están haciendo cada vez más crecientes. Por ende, se concluye que los problemas se vienen agravando día a día, lo que implica que la confiabilidad no es buena para el cliente.

\section{REFERENCIAS}

Báez, A. y Caraballo, R. (2004). Desarrollo de un plan de mantenimiento para una industria textil basado en mantenimiento productivo total utilizando un sistema computarizado (tesis de Ingeniería Mecánica). Universidad Central de Venezuela, Caracas.

Ballester, S., Olmed, P. y Tormos, B. (2002). El mantenimiento de las flotas de transporte. Técnica Industrial. 17(247). 
Chang, E. (2008). Propuesta de un modelo de gestión de mantenimiento preventivo para una pequeña empresa del rubro de minería para reducción de costo del servicio de alquiler (tesis para optar por el título de ingeniero industrial). Universidad Peruana de Ciencias Aplicadas, Lima.

Hernández, C. (1997). Metodología de la Investigación (1. a ed.). México: Mc Graw-Hill.

Hernández, M. (2011). Diseño de un plan de mantenimiento preventivo centrado en la confiabilidad de las máquinas retroexcavadoras de la empresa Solano y Asociados, C.A. de Porlamar estado Nueva Esparta (tesis de Ingeniería Mecánica). Instituto Universitario Politécnico Santiago Mariño Extensión Puerto Ordaz, Ciudad Guyana, Venezuela.

Herrera, M. (2010). Metodología e Implementación de un programa de gestión de mantenimiento. Ingeniería Industrial, 37(1).

Knezevic, J. (1996). Mantenimiento (1. ${ }^{a}$ ed.). España: Isdefe.

Martínez, F. (2013). Diseño de un plan de mantenimiento para un equipo de alta fiabilidad. Técnica Industrial. 20(301).

Mata, D., Aller, J. y Bueno, A. (2008). Análisis probabilístico del mantenimiento predictivo y correctivo de máquinas eléctricas rotativas en una planta trefiladora. Universidad, Ciencia $y$ Tecnología, 12(49).

Morales, J. (2012). Implantación de un programa de mantenimiento productivo total (TPM) al taller automotriz del I. municipio de Riobamba (IMR) (tesis de Ingeniería Automotriz). Escuela Superior Politécnica de Chimborazo, Riobamba, Ecuador.

Prando, R. (1996). Manual de gestión de mantenimiento a la medida (1. ${ }^{\mathrm{a}}$ ed.). San Salvador: Editorial Piedra Santa.

Rey, F. (2013). Elaboración y optimización de un plan de mantenimiento preventivo. Técnica Industrial. 20(308).

Rey, F. (2012). Determinación de la necesidad de un mantenimiento preventivo en una industria. Técnica Industrial. 20(300).

Torres, L. (2005). Mantenimiento. Su implementación y Gestión (2. a ed.). Argentina: Universitas. 
Vargas P. y Welder, U. (2004). Programa de mantenimiento para la maquinaria pesada de la zona vial 11, de caminos, en el Departamento de Izabal (tesis de Ingeniería Mecánica). Universidad de San Carlos de Guatemala.

Viveros, P., Stegmaier R., Kristjanpoller, F., Barbera, L. y Crespo, A. (2013). Propuesta de un modelo de gestión de mantenimiento y sus principales herramientas de apoyo. Ingeniare, 21(1).

Walpole R., Myers R. y Myers S. (1999). Probabilidad y estadística para ingenieros (6. ${ }^{\mathrm{a}}$ ed.). México: Pearson.

Zapata, C. (2009). Diseño de un sistema de gestión de mantenimiento preventivo para los equipos de planta $H$ y L II en la siderúrgica del Orinoco Alfredo Maneiro (tesis de Ingeniería Industrial). Universidad Nacional Experimental Politécnica Antonio José de Sucre, Puerto Ordaz, Venezuela. 